\title{
The Effect of Boattail Angles on the Near-Wake Structure of Axisymmetric Afterbody Models at Low-Speed Condition
}

\author{
The Hung Tran ${ }^{10}{ }^{1,2}$ \\ ${ }^{1}$ Institute of Theoretical and Applied Research, Duy Tan University, Hanoi 100000, Vietnam \\ ${ }^{2}$ Faculty of Aerospace Engineering, Le Quy Don Technical University, 236 Hoang Quoc Viet, Bac Tu Liem, Hanoi, Vietnam \\ Correspondence should be addressed to The Hung Tran; tranthehung1@duytan.edu.vn
}

Received 28 February 2020; Revised 21 June 2020; Accepted 23 July 2020; Published 17 August 2020

Academic Editor: Andre Cavalieri

Copyright (c) 2020 The Hung Tran. This is an open access article distributed under the Creative Commons Attribution License, which permits unrestricted use, distribution, and reproduction in any medium, provided the original work is properly cited.

\begin{abstract}
The effect of a boattail angle on the structure of the wake of an axisymmetric model was investigated at low-speed condition. Four conical boattail models with angles of $0^{\circ}$ (blunt-based body), $10^{\circ}, 16^{\circ}$, and $22^{\circ}$ were selected for this study. The Reynolds number based on the diameter of the model was around $1.97 \times 10^{4}$. Particle image velocimetry (PIV) was used to measure the velocity of the wake flow. The time-averaged flow characteristics including the length of recirculation of the afterbody, turbulent intensity, and Reynolds shear stress were analyzed and compared among those boattail models. The experimental results showed that the length of recirculation decreases with increasing boattail angle to $16^{\circ}$. At a boattail angle above $16^{\circ}$, the flow was fully separated near the shoulder and near-wake structure was highly changed. The turbulent intensity at a boattail angle of $22^{\circ}$ showed a similar level to that in the case of the blunt-based body. Flow behavior on boattail surface should be accounted as an important parameter affecting the wake width and drag of the model. Power spectral density and proper orthogonal decomposition (POD) analyses showed that a Strouhal number of $\mathrm{St}_{D}=0.2$ dominated for the boattail model up to $16^{\circ}$. The fully separated flow was dominated by a Strouhal number of $\mathrm{St}_{D}=0.03-0.06$, which was firstly presented in this study.
\end{abstract}

\section{Introduction}

An axisymmetric blunt-based body is featured by a large separation around the base. The high turbulent region behind the base causes a significant decrease in the base pressure and an increase in the base drag, noise, and structure fatigue [1]. As an example, the base drag could reach $50 \%$ of the total aerodynamic drag of the model at subsonic conditions [2]. Since the fossil fuel is quickly running out, reducing the base drag for energy saving is a very important task for aerodynamic researchers.

Boattail is an effective device to reduce base drag. A boattail model of $5^{\circ}$ could reduce the drag up to $21 \%$ in supersonic condition [3]. At low-speed condition, a conical boattail model of $15^{\circ}$ allows a reduced drag around 34\% [4]. Despite the simple design, flow behavior around the boattail is very complicated and is highly affected by its geometry. That flow behavior is characterized by a deceleration of the air near the base edge, separation at nonzero flow angle, and a large wake region behind the base [5-7]. Those charac- teristics are alternated for different boattail configurations and flow conditions such as the Mach number, Reynolds number, and boundary layer properties. Practically, the flow behavior of axisymmetric boattail cannot be predicted by theoretical analysis $[8,9]$.

Previously, Merz et al. [7], who investigated the nearwake of an axisymmetric semielliptical afterbody at lowspeed conditions, observed a significant decrease on the rear recirculation region comparing with that of the blunt-based body. They also showed that the wall curvature produces a large effect on the pressure drag and the flow just outside the shear layer is highly convex near the separation position. Howard and Goodman [10], who measured the drag of two boattail models of $10^{\circ}$ and $30^{\circ}$, indicated that the drag decreases largely at boattail models of $\beta=10^{\circ}$. At $\beta=30^{\circ}$, where fully separated flow was observed near the shoulder, the drag of the model is the same as the cases of the bluntbased body. However, the near-wake structure was not investigated in their study. Ilday et al. [4] who employed hot-wire devices to measure near-wake of the blunt-based body and 


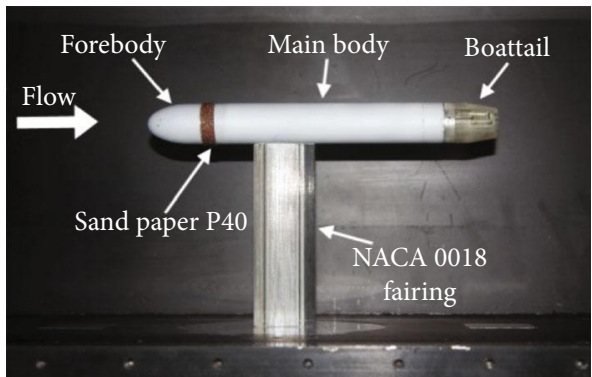

(a) Model in wind tunnel test

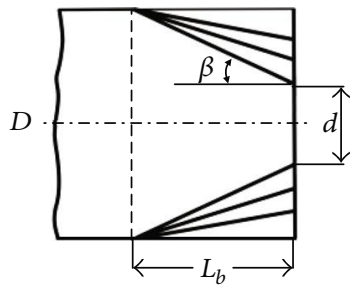

(b) Boattail configuration

FIGURE 1: Schematic picture of the experimental set-up.

TABle 1: Geometrical parameters of the boattails tested in this study.

\begin{tabular}{lcc}
\hline Angle $\beta\left(^{\circ}\right)$ & $d / D$ & $L_{b} / D$ \\
\hline 0 & 1.00 & 0.7 \\
10 & 0.70 & 0.7 \\
16 & 0.60 & 0.7 \\
22 & 0.43 & 0.7 \\
\hline
\end{tabular}

boattail model with an angle of $15^{\circ}$ observed a noticeable decrease in the turbulent intensity and length of recirculation behind the base. Mariotti et al. [6], who investigated contoured boattail models of $26.7^{\circ}$ and $40.4^{\circ}$, reported two flow types on the boattail surface, which are fully attached and separated conditions. Additionally, they showed that the turbulent intensity of the wake reduces with increasing boattail angle, whenever separation occurs on the boattail surface or not. Tran et al. [11], who conducted experiments conical boattails with a small change of angles from $10^{\circ}$ to $22^{\circ}$, observed three flow regimes on the boattail surface, which are attached flow, flow with separation bubble, and fully separated flow. However, the near-wake flow was not investigated in their study.

Despite the fact that many studies have been performed, previous studies mainly concentrated on the flow on the boattail surface and effect of the boattail angle on the drag of the model. In our knowledge, the near-wake structure was extracted for very limited boattail modes, mainly at angles of $26.7^{\circ}$ and $40.4^{\circ}$ [6]. Clearly, the wake structure of the boattail model at different flow conditions and its discrepancy with a blunt-based body was not totally understood.

The purpose of this study is to obtain clearly the effect of boattail angles on the structure of the wake flow. For investigations, two-component particle image velocimetry (PIV) was employed to measure instantaneous and time-averaged quantitative velocity fields on the vertical plane. Four conical boattail models with a fixed length and angles of $0^{\circ}$ (bluntbased body), $10^{\circ}, 16^{\circ}$, and $22^{\circ}$ were selected for this study. Time-averaged flow fields, turbulence levels, and Reynolds shear stress were analyzed and discussed in detail. The results show that flow behavior on the boattail surface is a very important parameter affecting the wake width and drag of the model. A proper orthogonal decomposition (POD) anal- ysis was performed to extract the large-scale features of the wake flow. Consequently, the different flow characteristic between the blunt-based body and boattail models was indicated clearly in this study. We also showed that the fully separated flow at the boattail model of $22^{\circ}$ was dominated by a Strouhal number of $\mathrm{St}_{D}=0.03-0.06$, which totally differs from the other boattail models.

\section{Experimental Set-Up}

2.1. Wind Tunnel Facility and Testing Model. The experiments were conducted in a low-speed wind tunnel with test section of $0.3 \times 0.3 \mathrm{~m}^{2}$ and a maximum velocity of $60 \mathrm{~m} / \mathrm{s}$ at Tohoku University, Sendai, Japan. The axisymmetric body was the same as the ones in previous studies of Tran et al. $([11,12])$. It has a diameter of $D=30 \mathrm{~mm}$ and a total length of $251 \mathrm{~mm}$. The blockage ratio of the model is around $0.8 \%$, which ensures the incompressible condition around the model.

The model is supported in test section by a strut with cross-section of NACA 0015 and maximum thickness of $8 \mathrm{~mm}$. The strut was placed at the front part of the main body to reduce its effect on the near-wake flow. Different conical afterbodies with the same length of $0.7 \mathrm{D}$ and boattail angles of $0^{\circ}, 10^{\circ}, 16^{\circ}$, and $22^{\circ}$ were added to the base by screw system. The boattail models were designed by a high-resolution $3 \mathrm{D}$ printer with a minimum layer thickness of $20 \mu \mathrm{m}$. Consequently, the connection between the main body and boattail is tight and their symmetric axes are coincided. The sketch of the model is shown in Figure 1. The parameters of the boattail models are listed in Table 1 , where $d$ is the diameter of the base and $L_{b}$ is the length of boattail.

Experiments were conducted at the free-stream velocity of $10 \mathrm{~m} / \mathrm{s}$. The turbulent intensity of the wind tunnel at this flow condition is less than $0.5 \%$. The corresponding baseradius Reynolds numbers is around $1.97 \times 10^{4}$. To generate the turbulent boundary layer on the body surface, a $10 \mathrm{~mm}$ wide sandpaper P20 with a mean diameter of $1 \mathrm{~mm}$ was placed at the forebody. The size of the sandpaper grit is referenced from an empirical method proposed by Braslow and Knox [13]. Here, the Reynolds number based on the thickness of the sandpaper is around $\operatorname{Re}=600$. It should be noted that the diameter of the particles' sandpaper is much more higher than that of the previous study by Rigas et al. [1], 


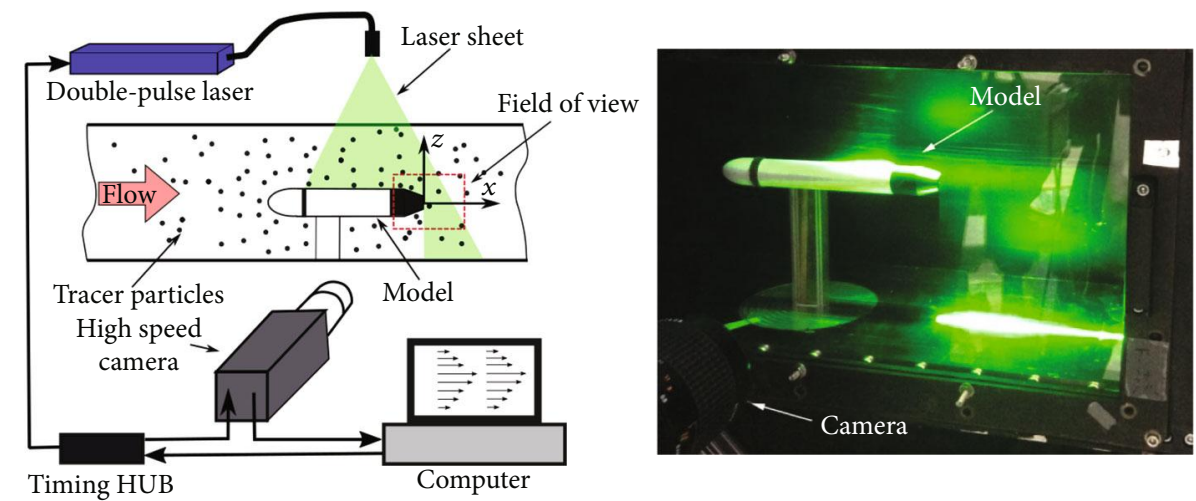

FIGURE 2: PIV set-up and model in experiments.

where emery paper grit P120 was used at a freestream velocity of $U_{\infty}=15 \mathrm{~m} / \mathrm{s}$.

The effect of strut support on the wake structure axisymmetric blunt-based body is a large problem at low-speed condition. Wolf et al. [14] indicated that a small change in the free-stream angle could lead to a change in the wake structure. However, this effect is reduced for the boattail model. Tran et al. [15], who conducted a free-levitated test and measurement with support system for axisymmetric boattail model at low-speed conditions, showed that the drag in the free-levitated test and drag using support system shows a similar trend for different boattail angles. Additionally, the effect of strut support on pressure distribution at lower and upper surfaces in the symmetric plane is the same. Consequently, the effect of strut support on the wake structure of the boattail model should be limited in this study. Since this study focuses on the effect of the boattail angle in the nearwake structure, we did not conduct measurement with freelevitated system. However, to obtain highly accurate data, further measurements in a free-levitated system and numerical scheme are required.

2.2. PIV Measurements. Two-component PIV system is employed to measure the velocity field over the boattail. The PIV set-up for velocity measurement is shown in Figure 2. A double-pulsed Nd-YLF Laser (LDY-303, Litron Lasers) was hung in the upper surface of the test section. The exposing length of the laser was $532 \mathrm{~nm}$, and the pulse duration was $150 \mathrm{~ns}$. The thickness of laser was setup at around $1 \mathrm{~mm}$, and time interval between the first and second images is fixed at $8 \mu \mathrm{s}$. The maximum movement of particles in images of a double frame was around 6 pixels, which is sufficient for the cross-correlation algorithm. To create luminescent particle for testing, a smoke generator LSG-500S was employed. The smoke generator has five lazkin nozzles and can provide air with smoke particles of around $1 \mu \mathrm{m}$ in diameter and $25 \mathrm{~m}^{3} / \mathrm{h}$ in volume. A high-speed camera Phantom v611 was placed on the side surface to capture the movement of particles. A lens of Nikkor $50 \mathrm{~mm}$ was attached to the camera. The resolution of the camera is $1280 \times 800$ pixels, and the exposure time was fixed at $1 \mu \mathrm{m}$. In this study, measurement was conducted at frequencies of $200 \mathrm{fps}$ and $2000 \mathrm{fps}$ to obtain static and unsteady behavior of the flow. The fields of view include $4 \mathrm{~mm}$ before the boattail shoulder and $73 \mathrm{~mm}$ after the trailing edge. The field of view is a rectangle region with dimensions of approximately $98 \times 61 \mathrm{~mm}^{2}$ providing resolution of 13 pixels $/ \mathrm{mm}$.

For data processing, the cross-correlation algorithm is applied. The detailed description of the algorithm indicated was proposed by Raffel et al. (2007) and Adrian and Westerweel (2011). Here, the first and second images are divided into small areas which are referred as interrogation windows. After that, the cross-correlations of each window in the first and the second images are calculated. The position of maximum cross-correlation shows the displacement of tracer particles in the second images. Since the time interval between the first and second images were known and displacement of interrogation windows was calculated, the velocity fields can be obtained. The formula for cross-correlation is shown as

$$
R(s)=\int_{W} I_{1}(X) I_{2}(X+s) d X
$$

where $I_{1}$ and $I_{2}$ represent the first and second images, $X$ is the coordinate, $W$ is the size of interrogation window, and $s$ is the displacement. As the velocity of each interrogation windows is obtained, velocity fields of the whole image could be constructed. The working principle of the PIV is shown in Figure 3. The method allows for obtaining the instantaneous velocity field from two images at different small times. By averaging instantaneous values at different time intervals, the mean velocity fields can be found.

In this study, the interrogation window is fixed at $8 \times 8$ pixels, which corresponds to an area of $0.65 \times 0.65 \mathrm{~mm}^{2}$. The process data was conducted by Dynamics Studio software. To reduce the noise and smoothen the results, several data processes, such as moving average validation and average filter, were also conducted as shown in Figure 4 . For each boattail model, a total of 2700 images were collected to build the instantaneous and time-averaged velocity fields.

\section{Results and Discussion}

3.1. Boundary-Layer Examination. The boundary-layer profile is examined for the blunt-based body at $23 \mathrm{~mm}$ before the base to obtain the initial characteristics of the separation 

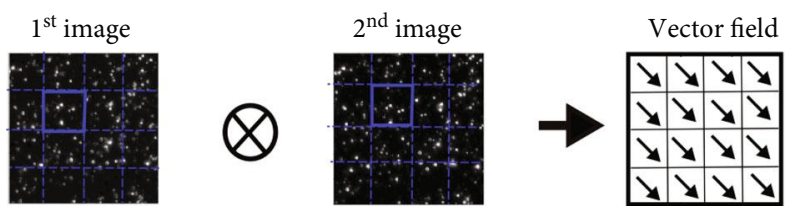

Figure 3: Cross-correlation algorithm for PIV measurement.

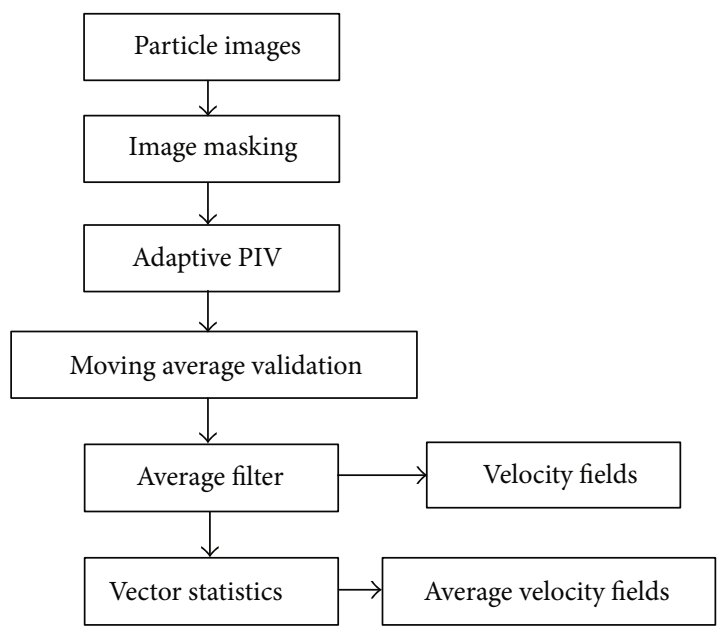

FIGURE 4: Process for calculating velocity vectors from PIV image.

flow. The results of boundary-layer profile are presented in Figure 5. Despite the fact that the number of points is limited, the clear boundary-layer profile is shown. The boundary thickness is calculated by the distance from the wall surface to a position where velocity reaches $95 \%$ of freestream velocity. The boundary profile is fitted well with $1 / 7^{\text {th }}$ law with the thickness around $6.8 \mathrm{~mm}$. Other parameters of the boundary layer including displacement thickness $\delta^{*}$, momentum thickness $\theta$, and shape factor $H$ are listed in Table 2. The shape factor is around $H=1.37$, which confirms that the boundary layer before the base is totally tripped into fully turbulent state. Consequently, the selection of roughness is sufficient for generating the turbulent boundary layer on the model surface.

3.2. Time-Averaged Flow Field. The streamwise velocity field mapped with streamlines in the vertical plane over the base is shown in Figure 6. Here, results measured at a frequency of $200 \mathrm{~Hz}$ were presented. The field of view is fixed at $3 D \times 1.2 D$, which corresponds to a resolution of $138 \times$ 55 points. The measurement time was around 13 seconds. It should be noted that the lowest Strouhal number for the blunt-based body is around $\mathrm{St}_{D}=0.002$, which corresponds to a time period of $1.3 \mathrm{~s}$ [1]. Consequently, the time measurement is sufficient for discussing the wake structure of the model. For the blunt-based body, the flow is bent around the base edge to form a recirculation region behind the base. The near-wake flow is characterized by a vortex ring with the center located at $(x / D, z / D)=(0.6, \pm 0.4)$. The flow is highly symmetric with respect to the centerline. The length of the recirculation region is around $1.18 D$. The distribution of near-wake is in highly agreement with a previous study of

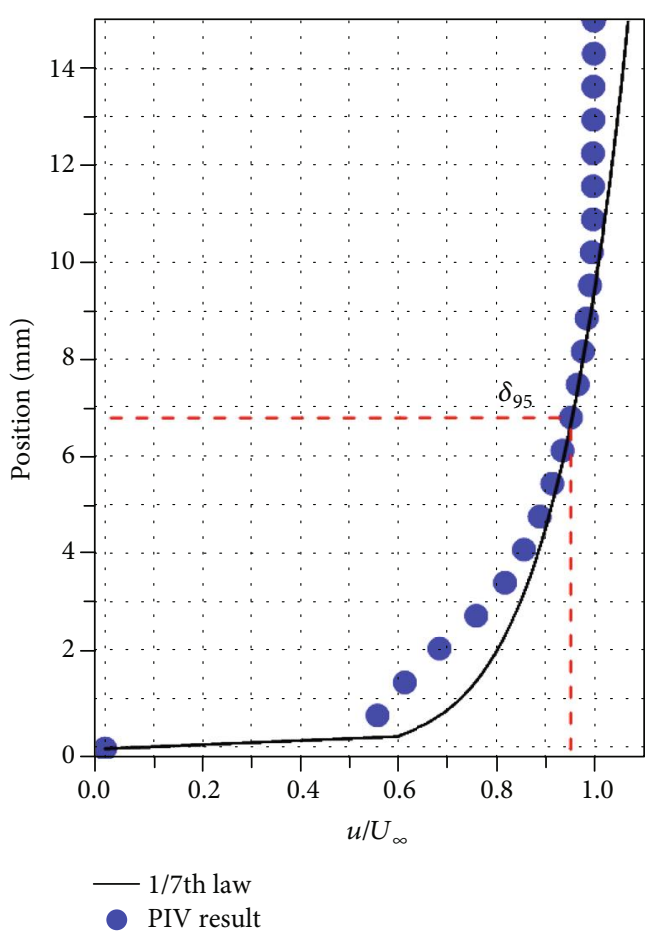

FIGURE 5: Boundary-layer profile of the blunt-based body.

TABLE 2: Characteristics of boundary layer.

\begin{tabular}{lc}
\hline Parameters & Value \\
\hline$\delta_{95} / D$ & 0.227 \\
$\delta^{*} / D$ & 0.0490 \\
$\theta / D$ & 0.0357 \\
$H$ factor & 1.37 \\
\hline
\end{tabular}

Wolf [16] at Reynolds number $\operatorname{Re}_{D}=4.5 \times 10^{5}$ and by Gentile et al. [17] at Reynolds number of $\operatorname{Re}_{D}=6.7 \times 10^{4}$. It is believed that the characteristic of flow over the blunt-based body does not change much in low-speed condition and at the Reynolds number between $10^{4}$ and $10^{6}$.

As the boattail angle is increasing from $\beta=0^{\circ}$ to $\beta=16^{\circ}$, the rear stagnation position moves close to the base edge, which shortens recirculation regions behind the base. The near-wake region becomes remarkably narrow by the boattail model. At boattail angle of $\beta=16^{\circ}$, a small reversed region is observed near the boattail shoulder. However, at $\beta=22^{\circ}$, the flow fully separates from the boattail shoulder and the whole boattail model is located inside the separation region. Those results are consistent with the observation of Tran et al. [11] at the Reynolds number around $\mathrm{Re}_{D}=4.34 \times 10^{4}$.

At the boattail angle higher than $0^{\circ}$, near-wake flow fields show unsymmetric with respect to the centerline $z /$ $D=0$. Our observation of the flow pattern at the boattail model of $22^{\circ}$ shows a similar behavior to the results by Mariotti et al. [6]. However, the results at boattail angles of $10^{\circ}$ and $16^{\circ}$ show a totally different trend. It is believed that the results are affected by the flow on the boattail surface and the strut support. Previously, Tran et al. (2019) 


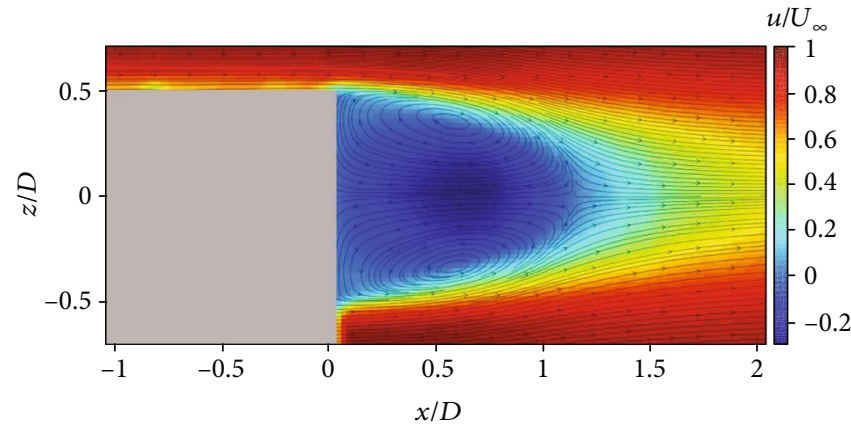

(a) $\beta=0^{\circ}$

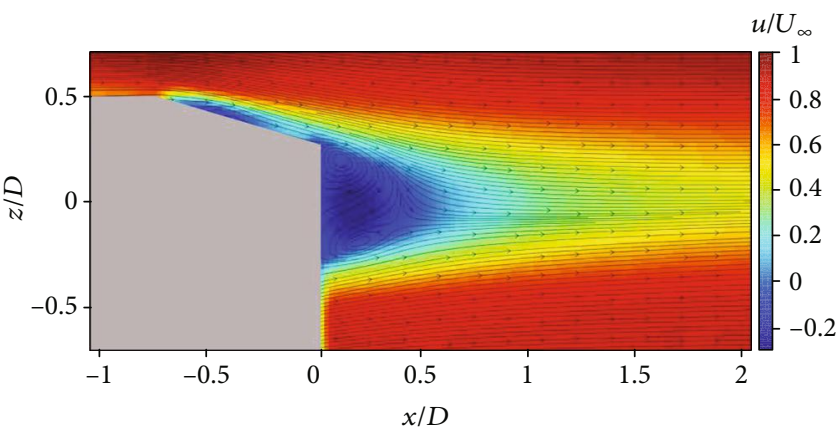

(c) $\beta=16^{\circ}$

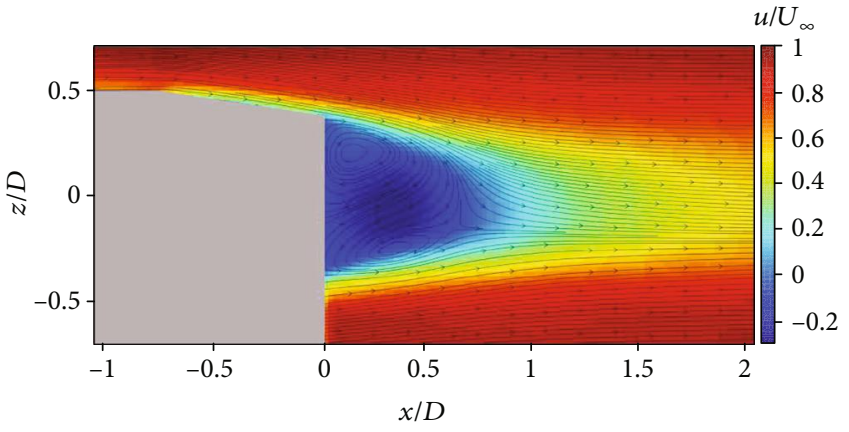

(b) $\beta=10^{\circ}$

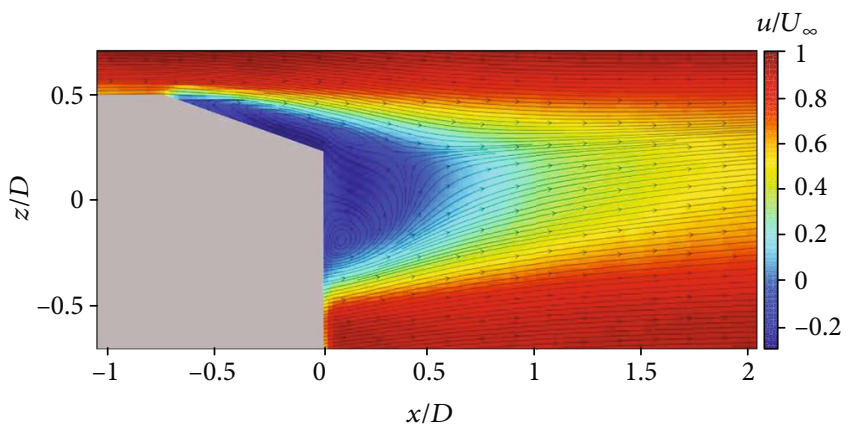

(d) $\beta=22^{\circ}$

Figure 6: Time-averaged streamline velocity magnitude at different boattail angles (a) $\beta=0^{\circ}$, (b) $\beta=10^{\circ}$, (c) $\beta=16^{\circ}$, and (d) $\beta=22^{\circ}$.

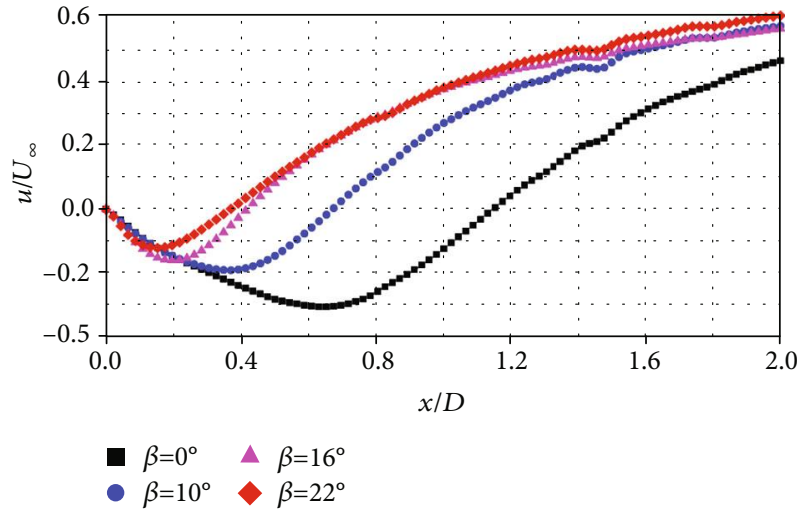

Figure 7: Velocity at the centerline.

show that strut support practically has no effect on pressure distribution on the base. Consequently, the effect of strut on the near-wake structure should be limited in this study. However, to obtain highly accurate data, further measurements in a free-levitated system and numerical scheme are required.

Figure 7 presents the distribution of streamwise velocity at the centerline for four boattail configurations. Clearly, the velocity magnitude in the near-wake region decreases with the boattail angle. At $\beta=0^{\circ}$, the maximum velocity reaches $0.35 U_{\infty}$ at around $x / D=0.65$. However, at $\beta=22^{\circ}$, the maximum velocity inside the wake reaches to approximately $0.13 U_{\infty}$ at $x / D=0.14$. Interestingly, even though the flow is fully separated at the boattail angle of $22^{\circ}$, the

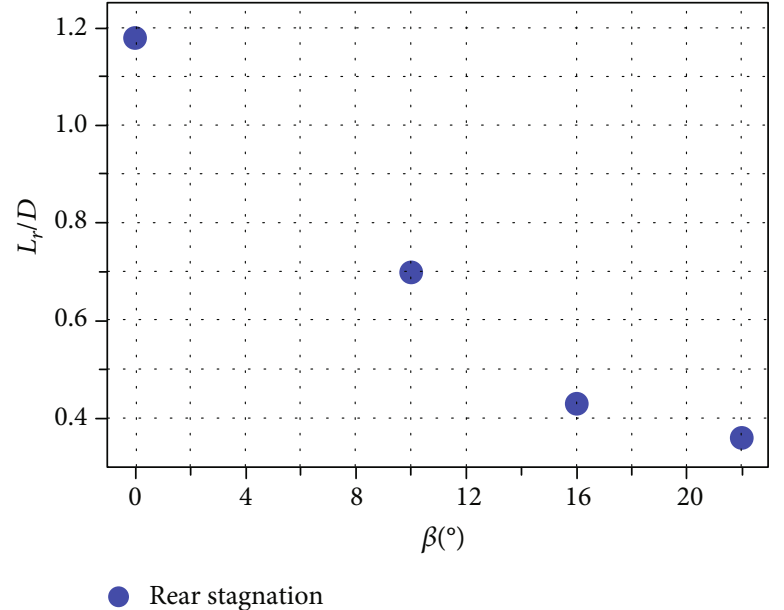

FIGURE 8: Location of rear stagnation position after body.

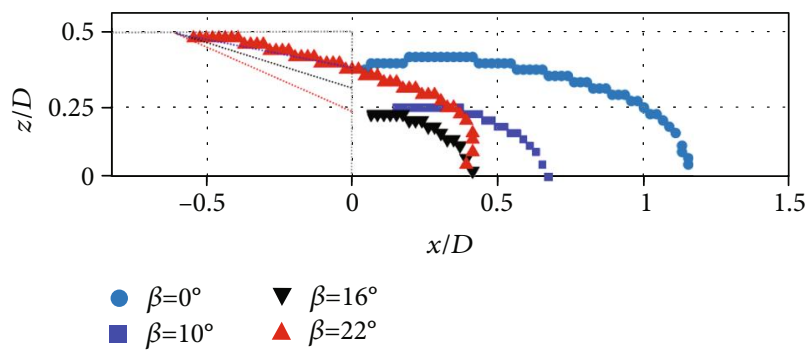

Figure 9: Lines with zero velocity of the wake flow. 


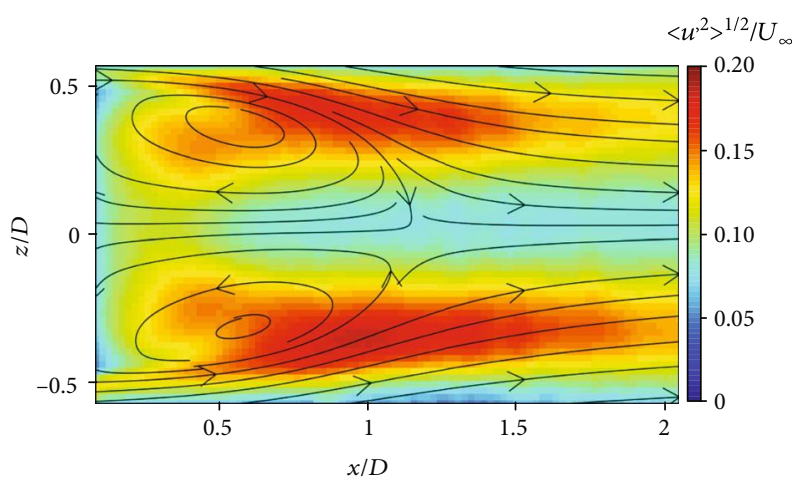

(a) $\beta=0^{\circ}$

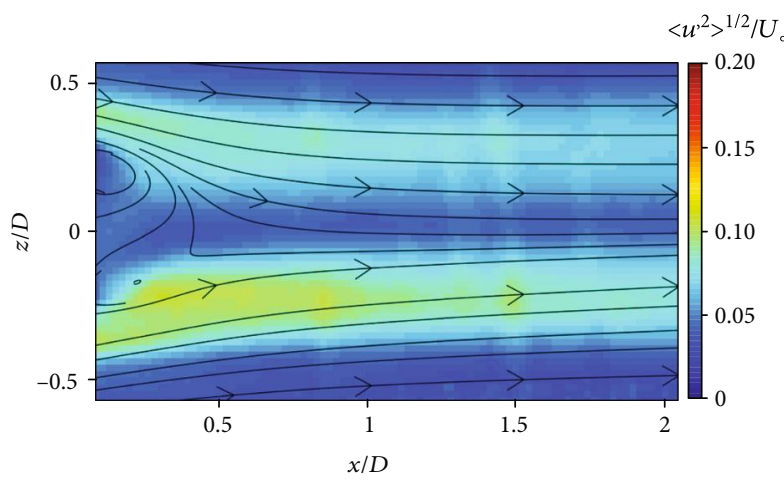

(c) $\beta=16^{\circ}$

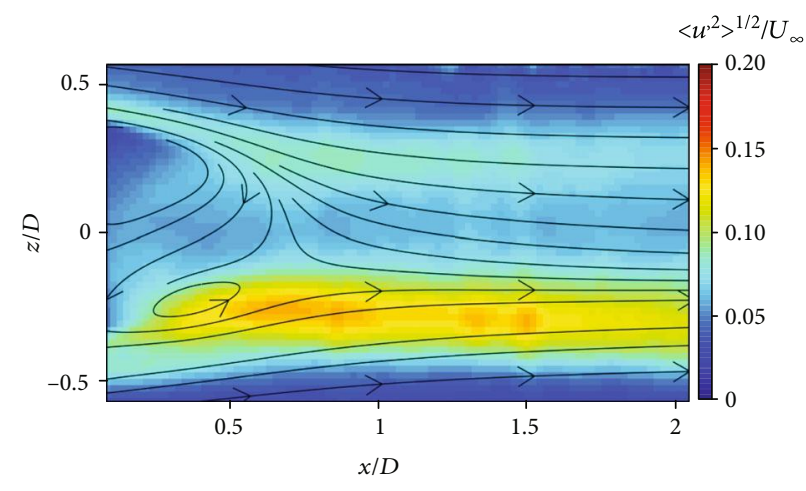

(b) $\beta=10^{\circ}$

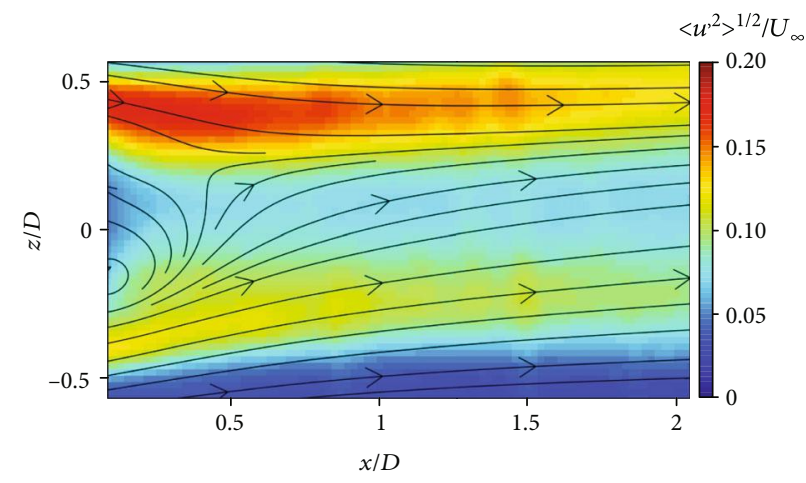

(d) $\beta=22^{\circ}$

Figure 10: Streamwise turbulence level $\left\langle\mathcal{u}^{2}>^{1 / 2}\right.$ at different boattail angles (a) $\beta=0^{\circ}$, (b) $\beta=10^{\circ}$, (c) $\beta=16^{\circ}$, and (d) $\beta=22^{\circ}$.

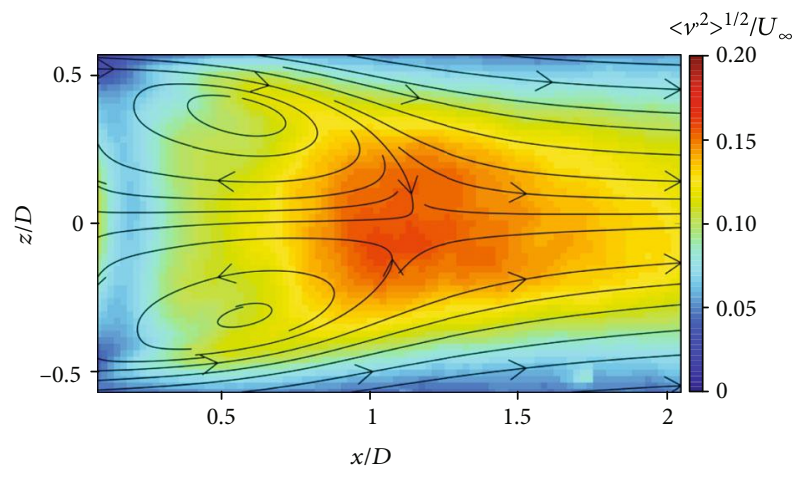

(a) $\beta=0^{\circ}$

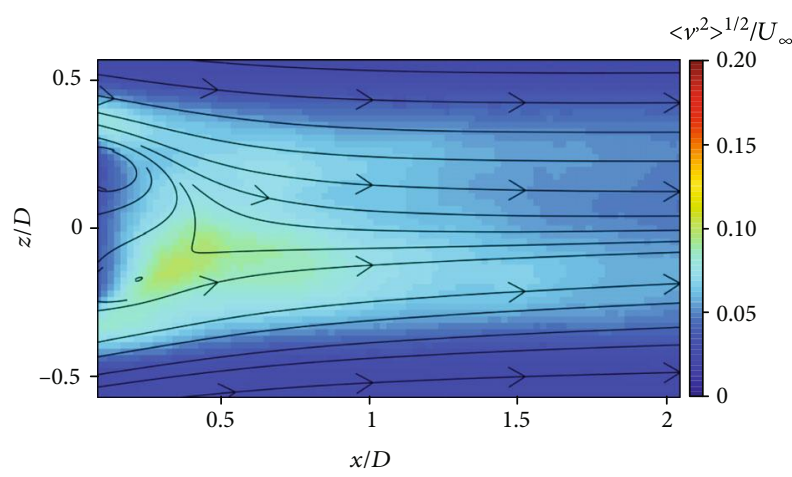

(c) $\beta=16^{\circ}$

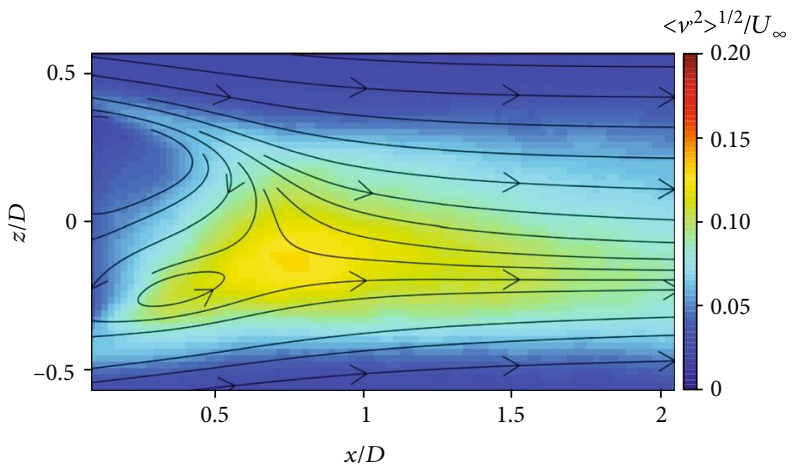

(b) $\beta=10^{\circ}$

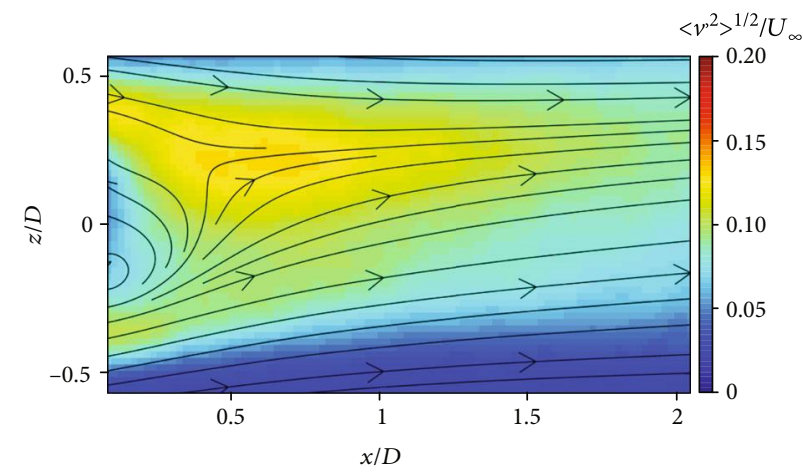

(d) $\beta=22^{\circ}$

Figure 11: Crosswise turbulence level $<v^{2}>^{1 / 2}$ at different boattail angles (a) $\beta=0^{\circ}$, (b) $\beta=10^{\circ}$, (c) $\beta=16^{\circ}$, and (d) $\beta=22^{\circ}$. 


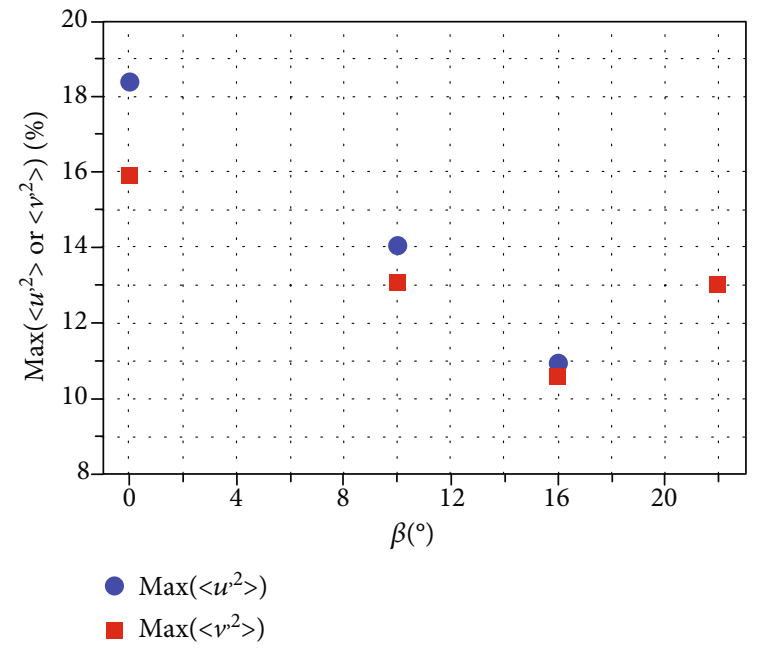

FIgURE 12: Maximum turbulence levels at different boattail angles.

velocity inside the wake is reduced. However, it should be noted that the wake region enlarges at that angle.

The location of the rear stagnation position as a function of the boattail angle is shown in Figure 8 while Figure 9 summarized the line with zero velocity in the near-wake region for four boattail models. Here, stagnation is determined by a position where the streamwise velocity in the centerline changes from negative to positive. Clearly, using boattail leads to a reduction of recirculation length afterbody. Additionally, the width of the near-wake region reduces with increasing the boattail angle from $0^{\circ}$ to $16^{\circ}$ (Figure 9). Comparing to the blunt-based body, the recirculation of the boattail model of $16^{\circ}$ decreases up to around 3 times $\left(L_{r} / D=0.43\right.$ ). Decreasing recirculation length is efficient for increasing the base pressure and thereby for reducing the base drag of the model. Ilday et al. [4], for example, showed that the length of recirculation afterbody decreases to $0.32 D$ for the boattail model of $15^{\circ}$. As a result, the base drag decreases from $C_{D}=0.32$ for the blunt-base model to $C_{D}=0.21$ for that boattail model. Tran et al. (2019), who measured the base drag by using pressure tap of different boattail configurations, observed a negative base drag at the boattail model of $16^{\circ}$. However, it should be noted that a significant different flow behavior near the base edge was reported for the blunt-base body and boattail models. As shown by Mariotti et al. [18], the streamline above the shear layer forms a convex curvature for the blunt-based body. The results lead to a very low pressure around the base surface and an increase in the drag of model. Additionally, it is seen that there is a linear relation between the length of recirculation and base drag of the model [18-20]. However, the flow behavior and relation between the recirculation length and base drag become complicated for the boattail model. In fact, the streamline above the shear layer of the boattail model forms a concave curvature. As a result, pressure recovery near the base is very high and the base drag decreases significantly. Interestingly, a sudden change of geometry near the shoulder generates a low-pressure region around conjunction of boattail and the main body (Tran et al., 2019). The low-pressure region creates an additional drag of the model. Consequently, for the boattail model, both base drag and boattail pressure drag should be considered.

We could see that, at a boattail angle of $22^{\circ}$, the flow is fully separated and all the boattail regions are inside the reversed flow region (Figure 9). As shown by Tran et al. (2019), the fully separated flow resulted in redistribution of pressure on the boattail surface. In fact, a low-pressure region is formed on the boattail and base surface and the drag of model increases again. Clearly, for the boattail model, the length of the recirculation region is not the main factor affecting the wake width and pressure drag. In that case, flow behavior on boattail surface should be considered. It should be noted that the static characteristics of near-wake structure was firstly presented and discussed in this study for a wide range of boattail models.

3.3. Statistical Turbulence Intensity. PIV results from different snapshot solutions allow calculation for turbulent characteristics of flow. In this section, the turbulence levels, which includes the streamwise turbulence level $\left\langle u^{\prime 2}\right\rangle^{1 / 2}$, crosswise turbulence level $<v^{\prime 2}>{ }^{1 / 2}$, and Reynolds number shear stress $\left\langle u^{\prime} v^{\prime}\right\rangle^{1 / 2}$, are calculated for understanding the statistical characteristics of the wake. The streamwise turbulence level is shown in Figure 10 for different boattail configurations. Clearly, similar patterns are observed for all models, where the highest streamwise turbulent intensity is observed in the free-shear layer region. The peak values occur before the rear stagnation position and are altered among those models. The streamwise turbulent intensity decreases from $18 \%$ at the blunt-based body to around $11 \%$ at the boattail model of $16^{\circ}$. It is believed that the decreasing mean velocity of the nearwake reduces the streamwise turbulent intensity when using the boattail model [21]. Additionally, the development of the boundary layer near the base edge, which leads to weaken of the free-shear layer when the boattail angle increases from $0^{\circ}$ to $16^{\circ}$, is the other reason for the decrease of streamwise turbulent intensity. At fully separated flow $\left(\beta=22^{\circ}\right)$, the streamwise turbulent intensity increases at around $17 \%$, which is similar to the case of the blunt-based body (Figure 10). Inherently, when the flow is fully separated, the wake region is enhanced. Those configurations, thereby, have practically no effect on drag reduction. The results of streamwise turbulent intensity in the current study differs to the finding of Mariotti [22] for the contour boattail model of $40.4^{\circ}$, where the flow separates on the boattail surface and turbulent intensity decreases comparing with the lower boattail angle.

In terms of the crosswise turbulence level, the peaks occur near the rear stagnation position, as shown in Figure 11. When the boattail model increases from $0^{\circ}$ to $16^{\circ}$, the peak values of the crosswise turbulence level decreases from around $16 \%$ to $11 \%$ (Figure 12 ). At the fully separated state, the crosswise turbulence level increases to approximately $13 \%$. Clearly, the turbulent characteristics are very sensitive with the boattail model. The distribution of turbulence levels shows a similar pattern with that over a circular cylinder, where Karman vortex shedding is the large-scale feature of the wake flow [23]. Additionally, it should be noted that the magnitude of peaks of streamwise turbulence levels is higher 


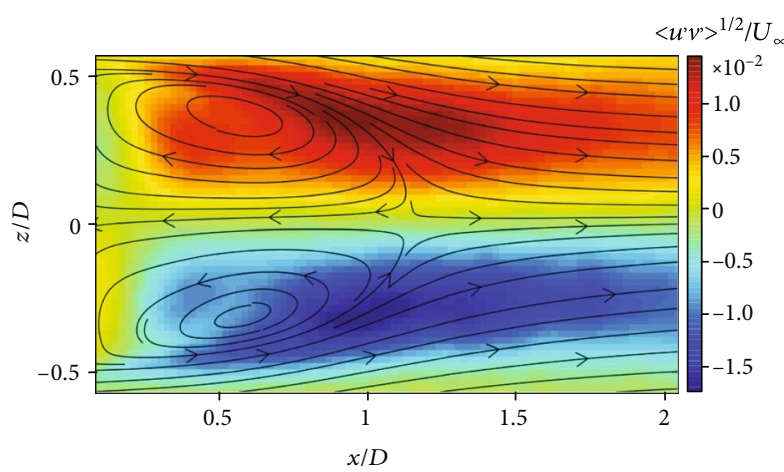

(a) $\beta=0^{\circ}$

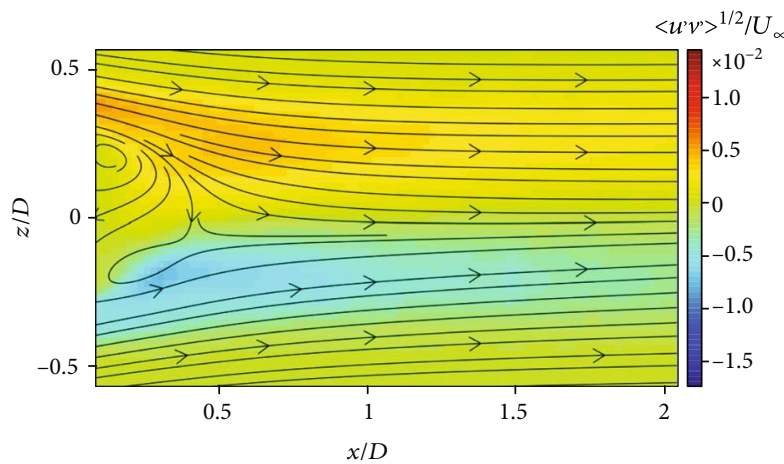

(c) $\beta=16^{\circ}$

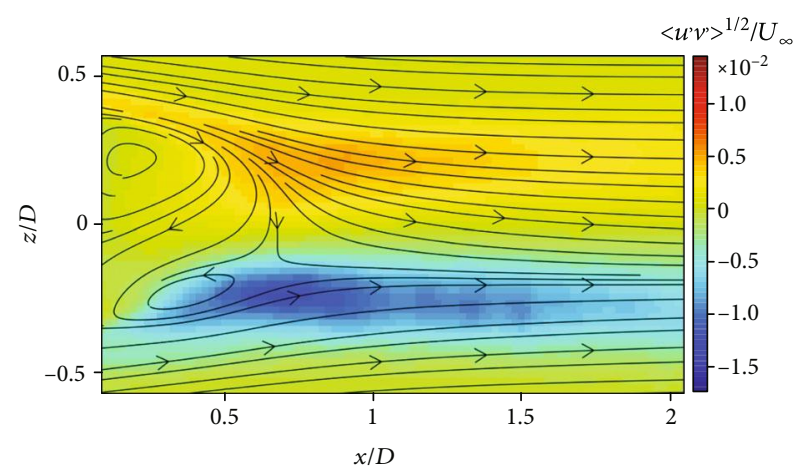

(b) $\beta=10^{\circ}$

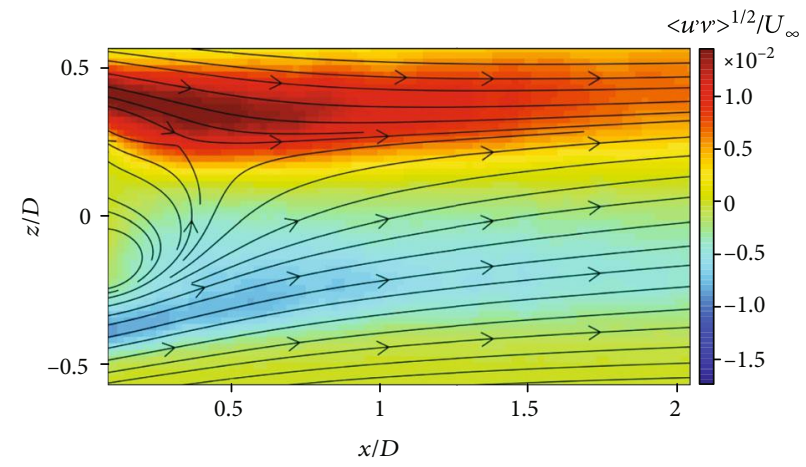

(d) $\beta=22^{\circ}$

Figure 13: Reynolds shear stress distribution (a) $\beta=0^{\circ}$, (b) $\beta=10^{\circ}$, (c) $\beta=16^{\circ}$, and (d) $\beta=22^{\circ}$.
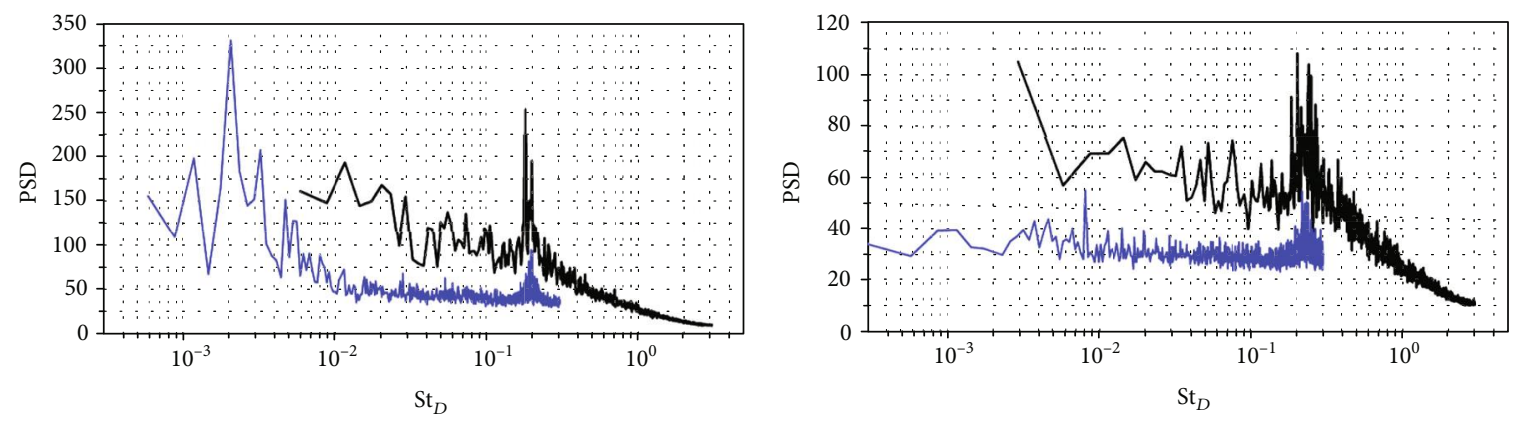

$-200 \mathrm{~Hz}$
$-2000 \mathrm{~Hz}$

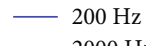

(a) $\beta=0^{\circ}$

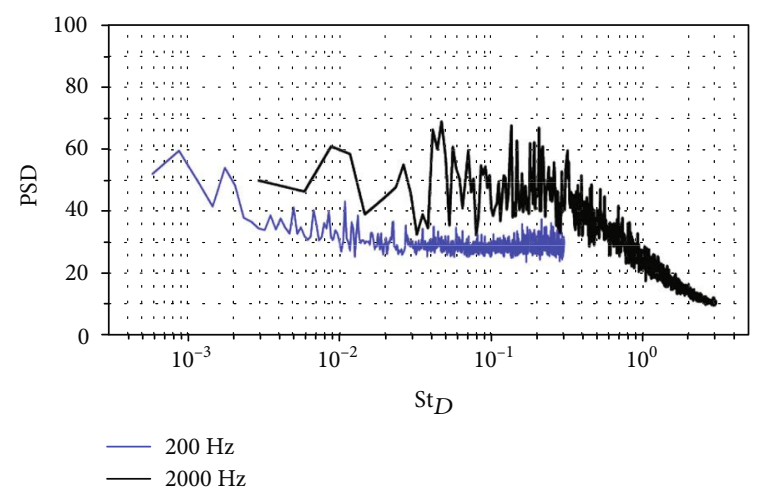

(c) $\beta=16^{\circ}$

(b) $\beta=10^{\circ}$

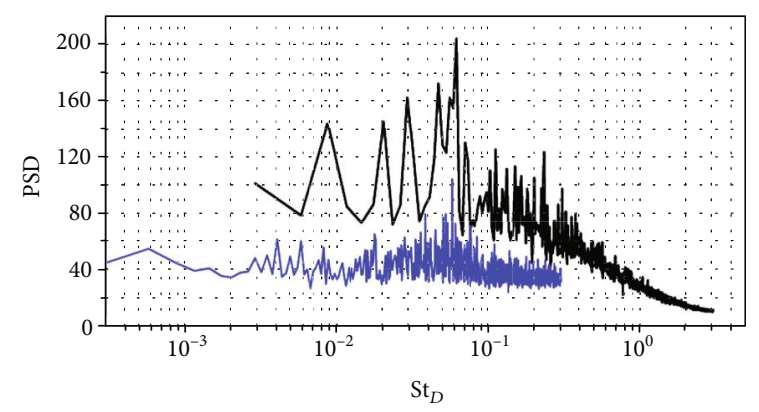

$-200 \mathrm{~Hz}$

(d) $\beta=22^{\circ}$

FigURE 14: Global power-spectral density of wake flow (a) $\beta=0^{\circ}$, (b) $\beta=10^{\circ}$, (c) $\beta=16^{\circ}$, and (d) $\beta=22^{\circ}$. 


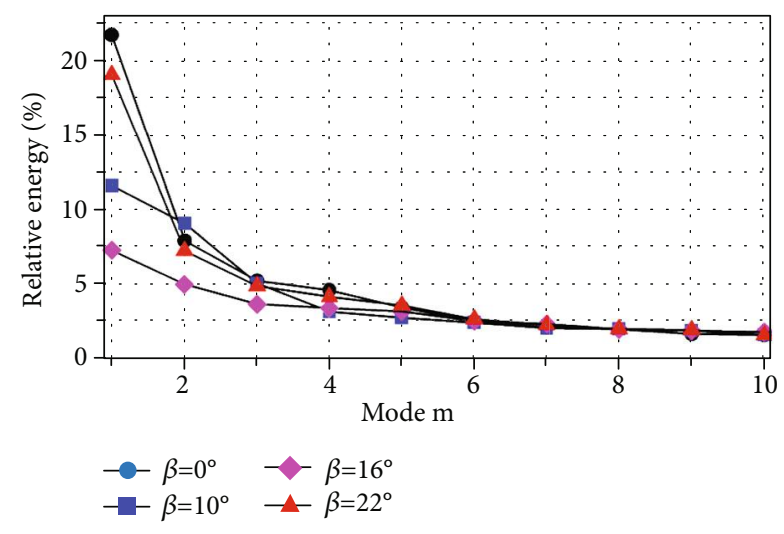

Figure 15: Relative energy of the first 10 POD modes.

than that of the crosswise component, which shows a strong influence on the turbulence intensity in axial direction.

The distribution of Reynolds shear stress is presented in Figure 13. It shows a similar pattern to the streamwise turbulent intensity. Consequently, the motion of free-shear layer is mainly impacted in the axial direction. The peak of Reynolds shear stress decreases when the boattail model increases from $0^{\circ}$ to $16^{\circ}$ and then increases at a boattail model of $22^{\circ}$.

3.4. Global Power Spectral Density of Streamwise Velocity. To obtain the main characteristics of flow fields, the global power spectral density of streamwise velocity is calculated. In this step, the power spectral density is calculated in each point and then averaged over the whole wake region. The global spectra, therefore, contain all frequencies of the flow fields [24]. The region for averaging is a rectangle with the width of $1.2 \mathrm{D}$ and the length of around $2.0 \mathrm{D}$ from the base surface. The results of that process are shown in Figure 14 for both measurements at frequencies of $200 \mathrm{~Hz}$ and $2000 \mathrm{~Hz}$. The Strouhal number $\mathrm{St}_{D}$ is calculated based on the diameter of the model.

Clearly, at $\beta=0^{\circ}$, the wake flow is dominated by a frequency of around $\mathrm{St}_{D}=0.2$ and a low frequency of $\mathrm{St}_{D}=$ 0.002 . Those spectral peaks are connected with the global oscillation of the vortex shedding which was well noted in previous studies by Wolf [16], Rigas et al. [1], and Gentile et al. [17]. The current study also observes a peak of the frequency in a range of $\mathrm{St}_{D}=0.01-0.06$, which are probably associated with the "bubble pumping" of the recirculation bubble [25]. A similar pattern is obtained for the boattail model of $\beta=10^{\circ}$, where the dominant flow mode occurs at frequencies between $\mathrm{St}_{D}=0.17-0.25$ (Figure 14). However, no dominant frequency was observed on the wake flow at $\beta=16^{\circ}$. It can be explained that the wake becomes small at that angle and the noise of measurement is high. To observe detailed flow behavior, POD will be applied in this study.

Interestingly, where the flow is fully separated at the boattail shoulder at $\beta=22^{\circ}$, the wake flow is dominated by frequencies between $\mathrm{St}_{D}=0.03-0.06$. The observation is in contrary to results observed by Mariotti [22], where the vortex shedding frequency increases with the boattail angle. In our knowledge, the above results were not presented before for the axisymmetric boattail model. Clearly, the main fea- ture of the near-wake is obtained from the global power spectral density.

3.5. Proper Orthogonal Decomposition of Velocity Data. To obtain the large-scale structure of wake flow and to analyze more details of the transient flow, POD analysis is conducted for velocity fields. In detail, the velocity values at time $t$ can be divided into averaged component and fluctuating component, which are written into the following equation:

$$
u(x, t)=\bar{u}(x, t)+u^{\prime}(x, t)
$$

The fluctuation component can be decomposed into modes by solving eigenvalues from a single value decomposition of the autocorrelation matrix $\mathrm{R}$ of the velocity fluctuation:

$$
R \phi_{k}=\lambda_{k} \phi_{k}
$$

where $R=\overline{u(x, t) \cdot u\left(x^{\prime}, t\right)}, \phi_{k}$ is the orthonormal function, which characterizes for mode $k$, and $\lambda_{k}$ indicates the relative energy distribution of that mode.

The most important feature of POD analysis is that it decomposes flow modes in descending order of relative energy distribution. Consequently, large-scale features of flow could be reconstructed. For details of this technique, readers can refer in Berkooz et al. [26]. In this study, the POD analyzed was performed in a rectangle region of $0.1 \leq$ $x / D \leq 2$ and $-0.6 \leq z / D \leq 0.6$ by using Matlab software. Additionally, only results at a measurement frequency of $2000 \mathrm{~Hz}$ were analyzed for comparisons of different boattail angles. The approach was similar to a previous study by Gentile et al. for axisymmetric back-step flow at lowspeed conditions.

The energy distribution of the first 10 POD modes is shown in Figure 15. As the boattail angle increases from $0^{\circ}$ to $16^{\circ}$, the relative energy of first mode decreases. At $\beta=0^{\circ}$, the first POD mode occupies around 21\% energy, while the relative energy of second and the third modes is less than $10 \%$. However, when the boattail model reaches to $\beta=16^{\circ}$, the energy of the first mode is around $7 \%$. It explains why no clear dominated mode was observed for the boattail model of $16^{\circ}$. Interestingly, at $\beta=22^{\circ}$, the high turbulence occurs on the near-wake region and the distribution of relative energy shows a similar feature in the case of the bluntbased body.

Figure 16 shows the spatial distribution and coefficient of the first mode $k=1$. For the case of $0^{\circ}$, the large-scale structure of wake flow is characterized by a single vortex with the core center located at $x / D=0.2$ behind the base. The first POD mode of the flow is associated with the flapping motion of the free-shear layer, which was well reported by Wolf [16] and Gentile et al. [17]. Gentile et al. [17] also showed that the flapping motion of the blunt-based body is characterized by a very low frequency in the range of $\mathrm{St}_{D}=10^{-3}-10^{-2}$ and a frequency of $\mathrm{St}_{D}=0.2$. A clear Strouhal number around $\mathrm{St}_{D}=$ 0.2 is illustrated in the current study. Additionally, a similar flow pattern was observed for other boattail models. Clearly, 

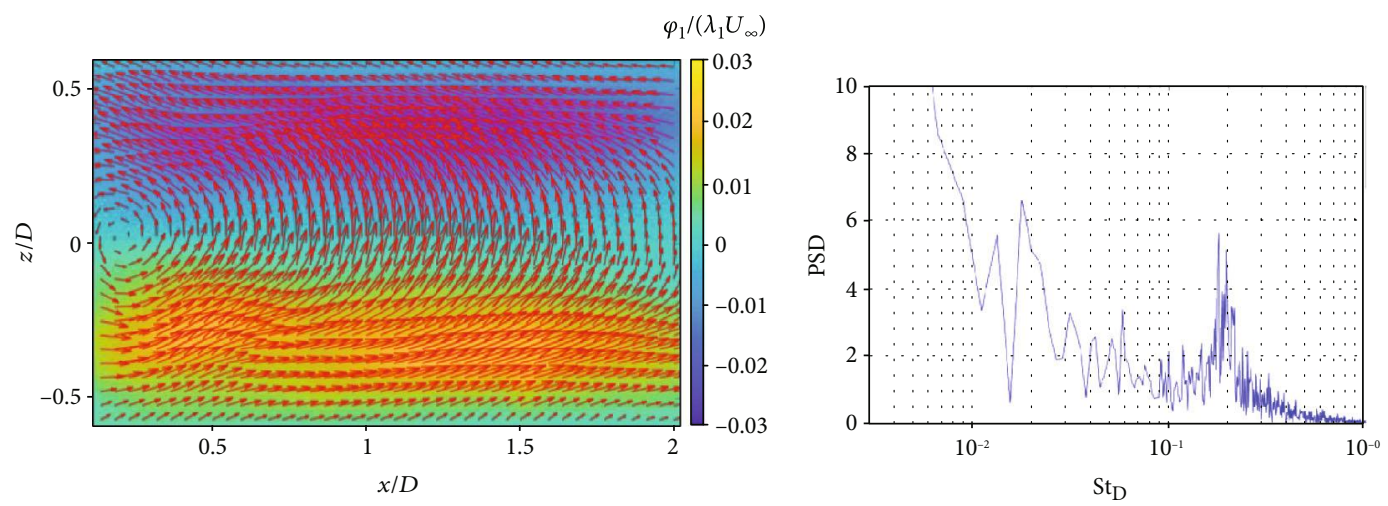

(a) $\beta=0^{\circ}$
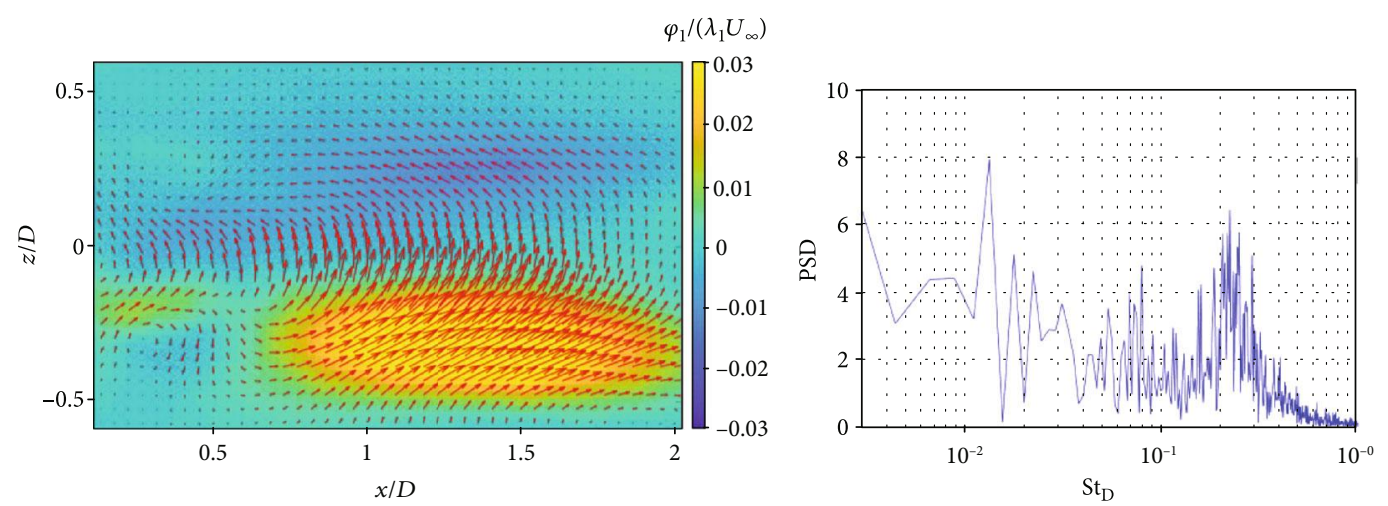

(b) $\beta=10^{\circ}$
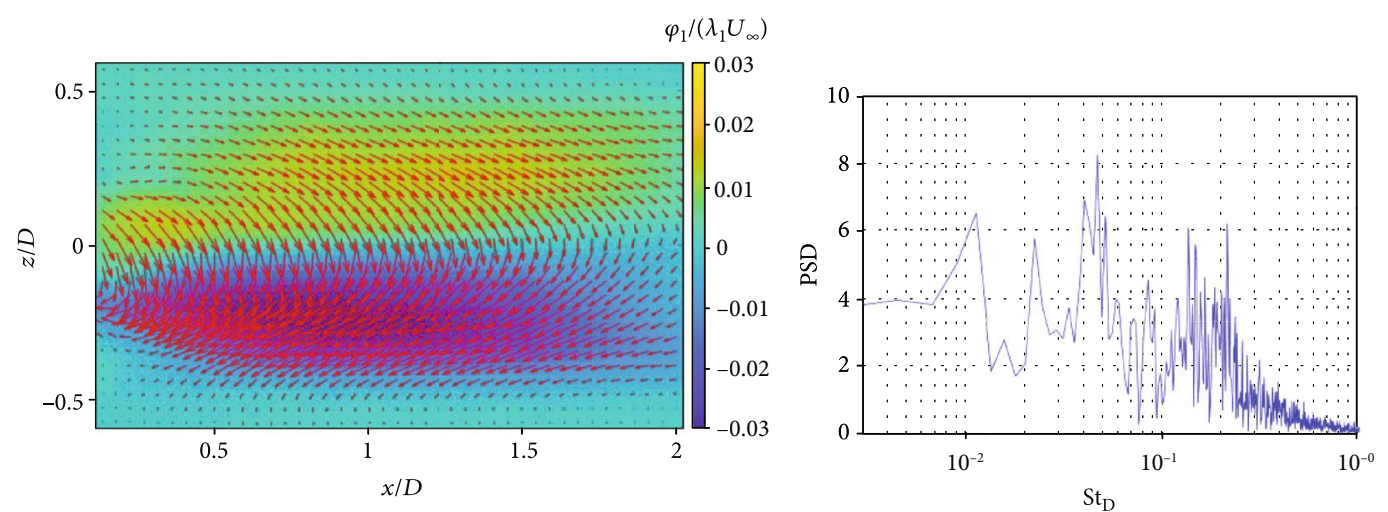

(c) $\beta=16^{\circ}$
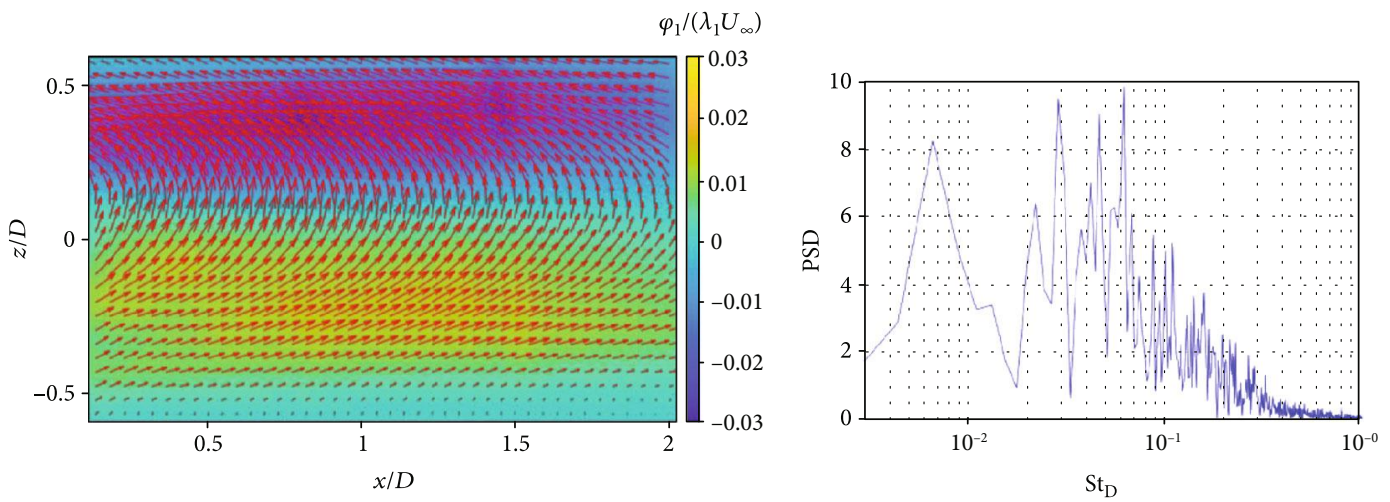

(d) $\beta=22^{\circ}$

FIgURE 16: Spatial distribution of the first POD mode at (a) $\beta=0^{\circ}$, (b) $\beta=10^{\circ}$, (c) $\beta=16^{\circ}$, and (d) $\beta=22^{\circ}$. 


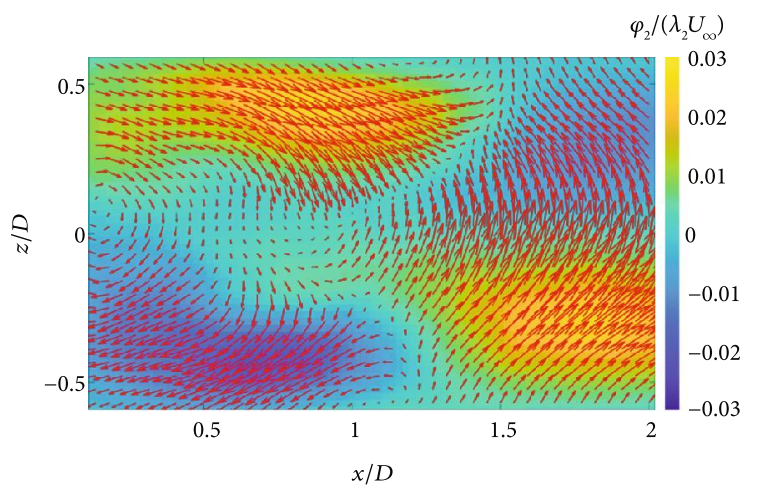

(a) $\beta=0^{\circ}$

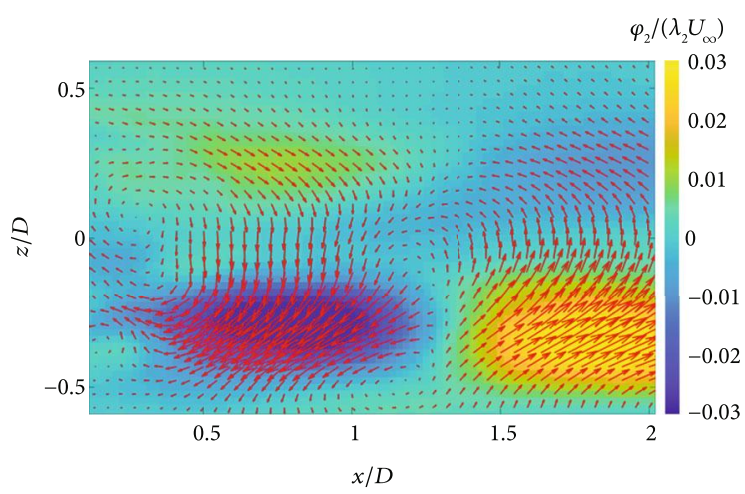

(b) $\beta=10^{\circ}$

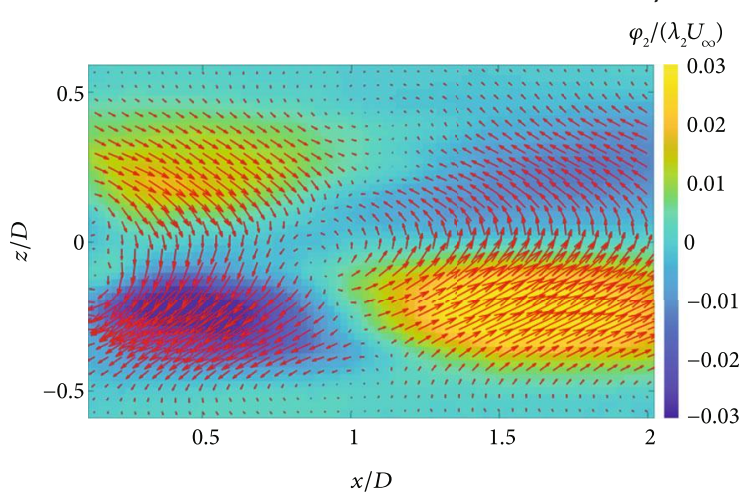

(c) $\beta=16^{\circ}$

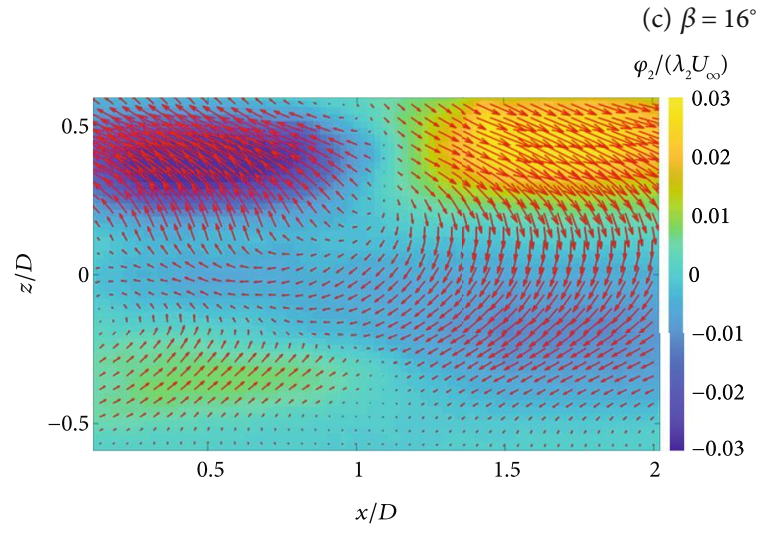

(d) $\beta=22^{\circ}$
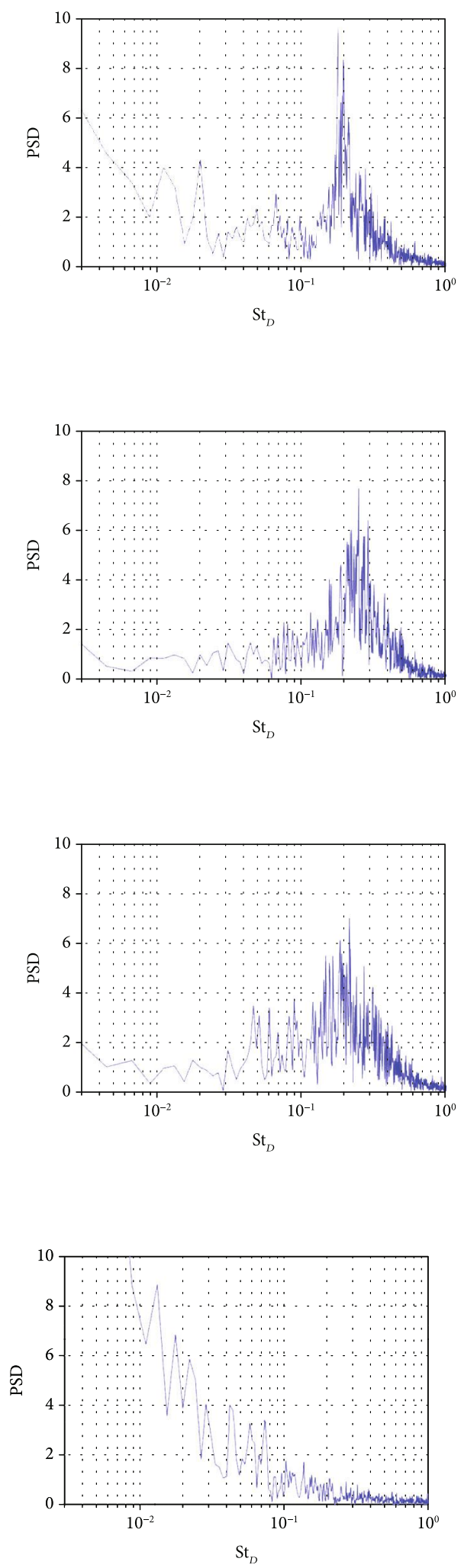

$\mathrm{St}_{D}$

FIgUre 17: Spatial distribution of the second POD mode at (a) $\beta=0^{\circ}$, (b) $\beta=10^{\circ}$, (c) $\beta=16^{\circ}$ and (d) $\beta=22^{\circ}$. 

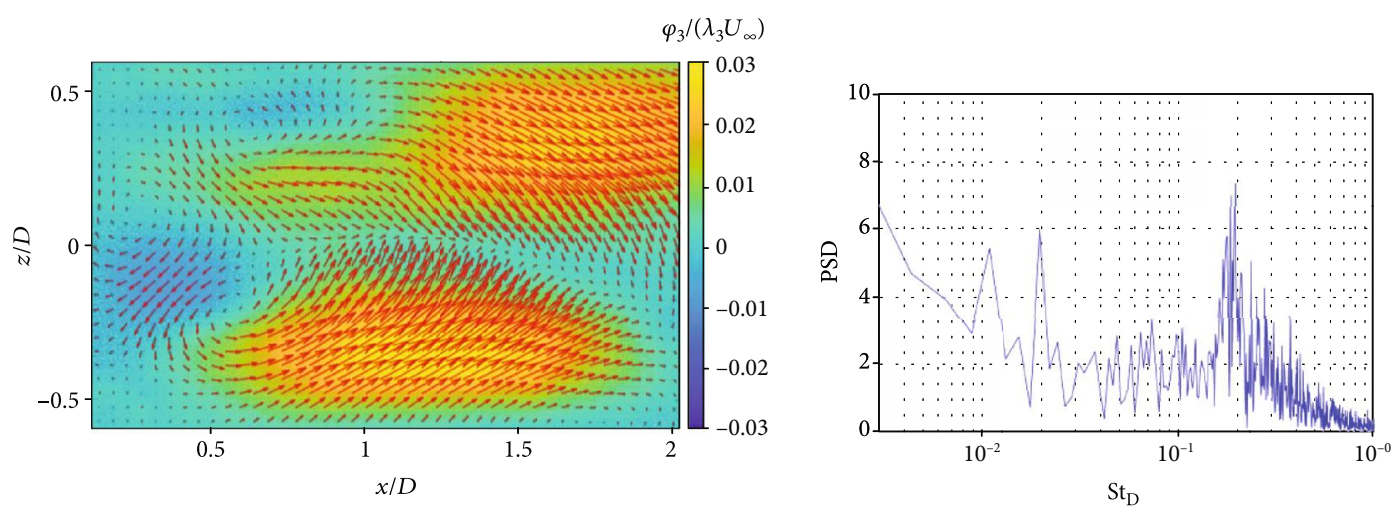

(a) $\beta=0^{\circ}$
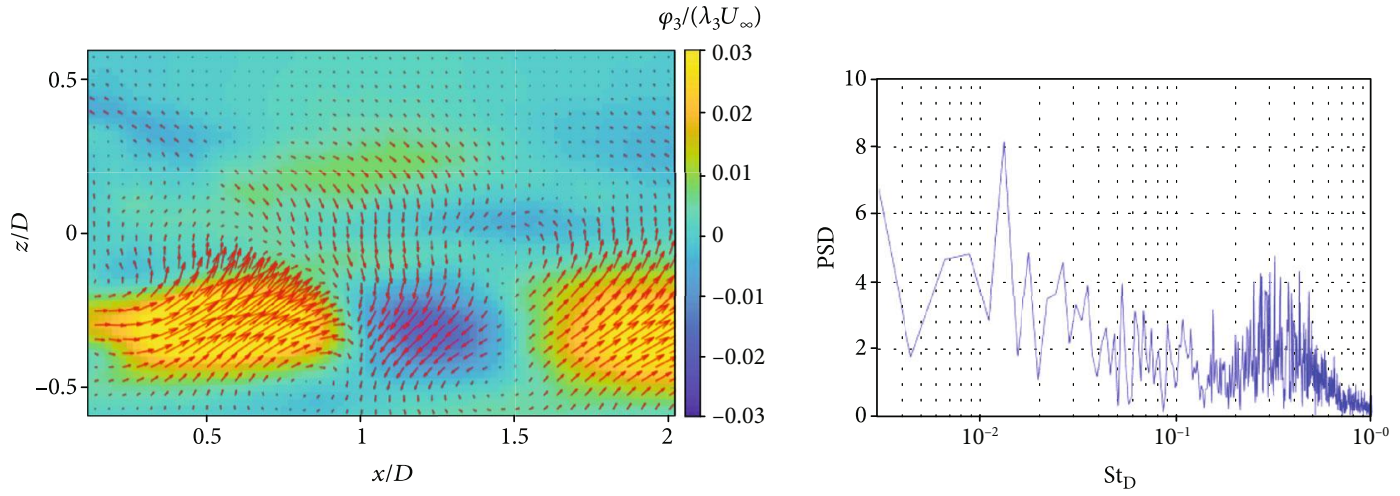

(b) $\beta=10^{\circ}$
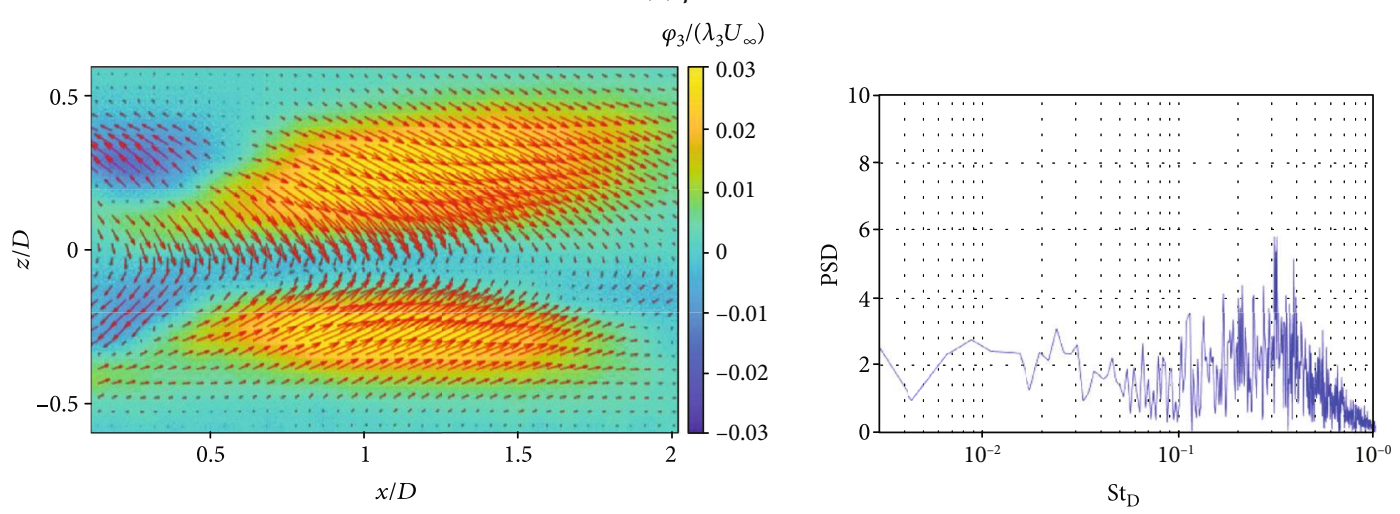

(c) $\beta=16^{\circ}$

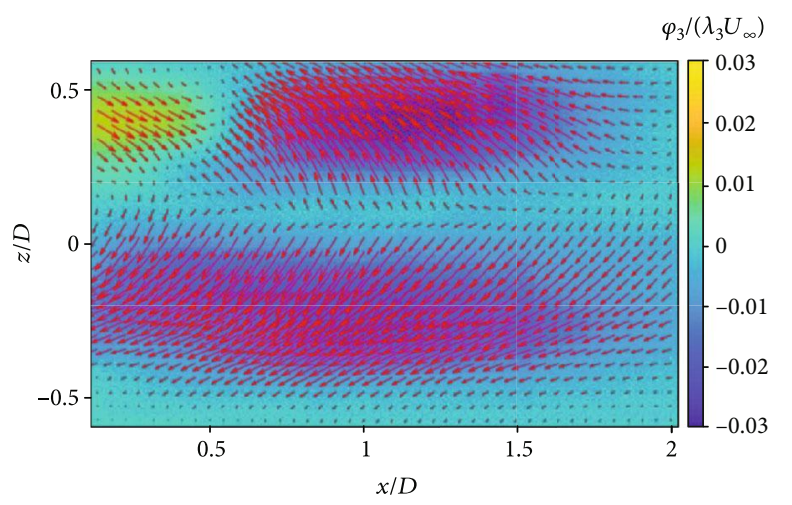

(d) $\beta=22^{\circ}$

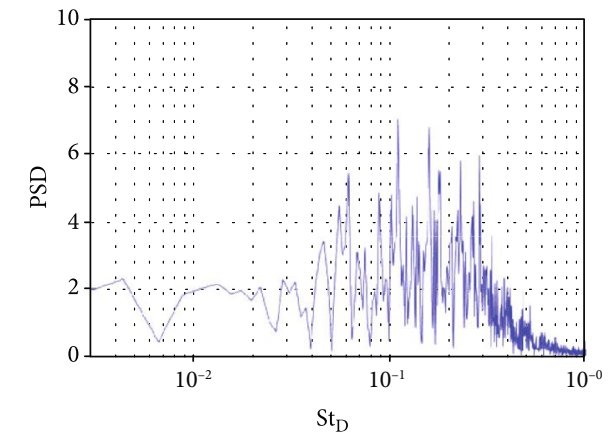

Figure 18: Spatial distribution of the third POD mode at (a) $\beta=0^{\circ}$, (b) $\beta=10^{\circ}$, (c) $\beta=16^{\circ}$, and (d) $\beta=22^{\circ}$. 
the flapping motion of the free-shear layer remains the largescale feature of the wake flow. However, as the boattail angle increases from $0^{\circ}$ to $16^{\circ}$, the region of the low and high intensity becomes narrower, which indicates that the effect of wake on the flow after the body decreases with increasing boattail angle. Interestingly, at the boattail model of $10^{\circ}$ and $16^{\circ}$, a Strouhal number of vortex shedding at around $\mathrm{St}_{D}=0.2$ was observed. However, at $\beta=22^{\circ}$, the vortex shedding is characterized by the Strouhal number around $\mathrm{St}_{D}=0.03-0.06$.

The spatial distribution and power spectral density for the time coefficient of the second POD mode $k=2$ are shown in Figure 17. At $\beta=0^{\circ}$, this structure shows smallscale of free-shear layer fluctuation which is characterized by low- and high-momentum regions with the length around 1.2D. This flow represents the "convective mode", which was reported by Gentile et al. [17]. Again, the Strouhal number of $\mathrm{St}_{D}=0.2$ is characterized for that model. A similar pattern is observed for other boattail configurations, where the scale length of changing momentum regions is from around $1 D$ to $1.2 D$. However, at $\beta=22^{\circ}$, no dominant frequency is observed.

The spatial distribution and power spectral density for the time coefficient of the third POD mode $k=3$ are illustrated in Figure 18. A similar pattern of power spectral density to those of mode $k=1$ is observed. However, the flow patterns for those boattail angles are changed. In fact, symmetric behavior could be observed clearly for boattail models of $\beta=0^{\circ}, 16^{\circ}$, and $22^{\circ}$. It is believed that this mode is the interference between vortex shedding and "bubble pumping" of the wake flow. Clearly, at low-speed conditions, the unsymmetric behavior dominates the flow structure for both blunt-based body and boattail models.

To summarize global power spectral density and POD analysis, we observed that the vortex shedding frequency at $S t D=0.2$ is the most dominant Strouhal number for boattail angles from $0^{\circ}$ to $16^{\circ}$. For the fully separated flow at $\beta=22^{\circ}$, the vortex shedding is still a large-scale dominant mode. However, the Strouhal number for that flow behavior is reduced to around $S t_{D}=0.03-0.06$. Our observation is totally different to the current results by Mariotti [22] for boattail angles of $0^{\circ}, 16.3^{\circ}$, and $40.4^{\circ}$, where the Strouhal number of vortex shedding increases with the boattail angle. In fact, in the previous study, the fully separated flow did not occur even for $\beta=40.4^{\circ}$. It is probably that the flow behavior on boattail surface is an important parameter, which affects the wake structure and the results of the Strouhal number.

\section{Conclusion}

In this study, the PIV technique is applied to measure the flow fields in the vertical plane of axisymmetric boattail models at low-speed conditions. Four boattail angles of $0^{\circ}$, $10^{\circ}, 16^{\circ}$, and $22^{\circ}$ were tested at the Reynolds number around $1.97 \times 10^{4}$ based on the model diameter. The results of the blunt-based body are highly consistent with those of previous studies. Major conclusions of the study are as follows.
4.1. Steady Behavior. The boattail model has a large effect in the near-wake structure. As the angle increases from $0^{\circ}$ to $22^{\circ}$, the length of recirculation decreases from around 1.18 $D$ to $0.36 D$ and the maximum velocity inside the wake region decreases from $0.35 U_{\infty}$ to $0.13 U_{\infty}$. At the boattail model below $16^{\circ}$, the flow is separated near the base edge. However, at $\beta=22^{\circ}$, the flow is fully separated at the shoulder and the whole boattail is located inside the separation region. The width of the near-wake is enlarged at that boattail angle.

The turbulent intensity afterbody decreases when the boattail angle increases from $0^{\circ}$ to $16^{\circ}$. At fully separated flow $\left(\beta=22^{\circ}\right)$, the peak value of the streamwise turbulence level is the same as the case of the blunt-based body. The peak value of the crosswise turbulent intensity slightly increases comparing with the boattail model of $16^{\circ}$. The length of the recirculation region is not the main factor affecting the wake width, drag, and other wake characteristics of the model. The flow behavior on boattail surface should be accounted as an important parameter affecting those factors at lowspeed conditions.

4.2. Unsteady Behavior. The analyses of global power spectral density and POD modes show that when the flow attached on boattail surface is at $\beta \leq 16^{\circ}$, the near-wake is dominated by a Strouhal number of $\mathrm{St}_{D}=0.2$. The energy for that flow mode decreases when the boattail model grows up from $0^{\circ}$ to $16^{\circ}$. At the boattail model of $22^{\circ}$, the wake flow is characterized by frequencies in a range of $\mathrm{St}_{D}=0.03-0.06$ and the distribution of relative energy is similar to the case of the blunt-based body. Although symmetric flow occurs at the third mode $k=3$ for some boattail models, the vortex shedding is the most dominated flow pattern at low-speed conditions. Our results of vortex shedding frequency for boattail models of $10^{\circ}, 16^{\circ}$, and $22^{\circ}$ are contracted with previous observation for the axisymmetric boattail model and are firstly presented in this study.

\section{Data Availability}

The data was processed by Tran The Hung at Duy Tan Univerisy and Le Quy Don Technical University.

\section{Conflicts of Interest}

None of the authors have any conflicts of interest.

\section{Acknowledgments}

The author would like to thank Professor Keisuke Asai and Professor Taku Nonomura at the Department of Aerospace Engineering, Tohoku University, in Japan for their support during the experimental process.

\section{References}

[1] G. Rigas, A. R. Oxlade, A. S. Morgans, and J. F. Morrison, "Low-dimensional dynamics of a turbulent axisymmetric wake," Journal of Fluid Mechanics, vol. 755, p. R5, 2014. 
[2] R. J. Krieger and S. R. Vukelich, "Tactical missile drag, tactical missile aerodynamics," Progress in Astronautics and Aeronautics, vol. 104, pp. 383-420, 1986.

[3] J. L. Herrin and J. C. Dutton, "Supersonic near-wake afterbody boattailing effects on axisymmetric bodies," Journal of Spacecraft and Rockets, vol. 31, no. 6, pp. 1021-1028, 1994.

[4] O. Ilday, H. Acar, M. K. Elbay, and V. Atli, "Wakes of three axisymmetric bodies at zero angle of attack," AIAA Journal, vol. 31, no. 6, pp. 1152-1154, 1993.

[5] G. Buresti, G. V. Iungo, and G. Lombardi, "Method for the drag reduction of bluff bodies and their application to heavy road-vehicles," DDIA 10-2007, 1st Interim Report Contract between CRF and DIA, 2007.

[6] A. Mariotti, G. Buresti, G. Gaggini, and M. V. Salvetti, "Separation control and drag reduction for boat-tailed axisymmetric bodies through contoured transverse grooves," Journal of Fluid Mechanics, vol. 832, pp. 514-549, 2017.

[7] R. A. Merz, C. H. Yi, and C. E. G. Przirembel, "The subsonic near-wake of an axisymmetric semielliptical afterbody," AIAA Journal, vol. 23, no. 10, pp. 1512-1517, 1985.

[8] W. A. Mair, "Drag-reducing techniques for axisymmetric bluff bodies," in Aerodynamic Drag Mechanisms of Bluff Bodies and Road Vehicles, G. Sovran, T. Morel, and W. T. Mason, Eds., General Motors Research Laboratories, Plenum Press, New York, 1978.

[9] P. R. Viswanath, "Flow management techniques for base and afterbody drag reduction," Progress in Aerospace Sciences, vol. 32, no. 2-3, pp. 79-129, 1996.

[10] F. G. Howard and W. L. Goodman, "Axisymmetric bluff-body drag reduction through geometrical modification," Journal of Aircraft, vol. 22, no. 6, pp. 516-522, 1985.

[11] T. H. Tran, T. Ambo, T. Lee, L. Chen, T. Nonomura, and K. Asai, "Effect of boattail angles on the flow pattern on an axisymmetric afterbody surface at low speed," Experimental Thermal and Fluid Science, vol. 99, pp. 324-335, 2018.

[12] T. H. Tran, T. Ambo, T. Lee et al., "Effect of Reynolds number on flow behavior and pressure drag of axisymmetric conical boattails at low speeds," Experiments in Fluids, vol. 60, no. 3, 2019.

[13] A. L. Braslow and E. C. Knox, "Simplified methods for determination of critical height of distributed roughness particles for boundary-layer transition at Mach numbers from 0 to 5," Technical Note No 4363, National Advisory Committee for Aeronautics NASA, Washington, 1958.

[14] C. C. Wolf, Y. You, R. Hörnschemeyer, H. Lüdeke, and V. Hannemann, "Base-flow sensitivity of a generic rocket forebody towards small freestream angles," Progress in Flight Physics, P. Reijasse, D. Knight, M. Ivanov, and I. Lipatov, Eds., vol. 5, pp. 169-190, 2013.

[15] T. H. Tran, T. Ambo, L. Chen, T. Nonomura, and K. Asai, "Effect of Boattail Angle on Pressure Distribution and Drag of axisymmetric afterbodies under low-speed Conditions," Transactions of The Japan Society For Aeronautical and Space Sciences, vol. 62, no. 4, pp. 219-226, 2019.

[16] C. C. Wolf, The subsonic near-wake of bluff bodies, [Ph.D. thesis], Institute of Aeronautics and Astronautics, RWTH Aachen, Germany, 2013.

[17] V. Gentile, F. F. J. Schrijer, B. W. van Oudheusden, and F. Scarano, "Afterbody effects on axisymmetric base flows," AIAA Journal, vol. 54, no. 8, pp. 2285-2294, 2016.
[18] A. Mariotti, G. Buresti, and M. V. Salvetti, "Connection between base drag, separating boundary layer characteristics and wake mean recirculation length of an axisymmetric blunt-based body," Journal of Fluids and Structures, vol. 55, pp. 191-203, 2015.

[19] D. Barros, J. Boree, B. R. Noack, A. Spohn, and T. Ruiz, "Bluff body drag manipulation using pulsed jets and coanda effect," Journal of Fluid Mechanics, vol. 805, pp. 422-459, 2016.

[20] M. Grandemange, M. Gohlke, and O. Cadot, "Bi-stability in the turbulent wake past parallelepiped bodies with various aspect ratios and wall effects," Physics of Fluids, vol. 25, no. 9, article 095103, 2013.

[21] V. Atli, "Wakes of four complex bodies of revolution at zero angle of attack," AIAA Journal, vol. 27, no. 6, pp. 707-711, 1989.

[22] A. Mariotti, "Axisymmetric bodies with fixed and free separation: Base-pressure and near- wake fluctuations," Journal of Wind Engineering and Industrial Aerodynamics, vol. 176, no. 2018, pp. 21-31, 2018.

[23] R. Perrin, M. Braza, E. Cid et al., "Near-wake turbulence properties in the high Reynolds number incompressible flow around a circular cylinder measured by two- and threecomponent PIV," Flow, Turbulence and Combustion, vol. 77, no. 1-4, pp. 185-204, 2006.

[24] J. D'Adamo, R. Godoy-Diana, and J. E. Wesfreid, "Spatiotemporal spectral analysis of a forced cylinder wake," Physical Review E, vol. 84, no. 5, article 056308, 2011.

[25] E. Berger, D. Scholz, and M. Schumm, "Coherent vortex structures in thewake of a sphere and a circular disk at rest and under forced vibrations," Journal of Fluids and Structures, vol. 4, no. 3, pp. 231-257, 1990.

[26] G. Berkooz, P. Holmes, and J. L. Lumley, "The proper orthogonal decomposition in the analysis of turbulent flows," Annual Review of Fluid Mechanics, vol. 25, no. 1, pp. 539-575, 1993. 